Res Nondestr Eval (1992) 4:37-54

(C) 1992 Springer-Verlag New York Inc.

\title{
A Knowledge-Based System for Nondestructive Testing of Polymeric Composite Components
}

\author{
Enio Gomes ${ }^{*}$ and Sridhar Kota ${ }^{2}$ \\ ${ }^{1}$ Ford Motor Company, Dearborn, Michigan; 2 Department of Mechanical Engineering and \\ Applied Mechanics, The University of Michigan, Ann Arbor, MI 48109-2125, USA.
}

\begin{abstract}
This paper presents a formalization of the knowledge domain of nondestructive quality control of polymeric composite components. The formalization scheme presented in this paper has been implemented in a prototype knowledge-based expert system (KBES), called NICC for nondestructive inspection of composite components, to help in the quality assurance of these parts. Geometric and bonding characteristics of individual and assembled components are taken into account, as opposed to the better understood evaluation of well-behaved test specimens. The use of nondestructive techniques in the inspection of plastic and polymeric composites is fairly recent and hence, the knowledge required to develop a KBES is still very scattered and not yet fully covered in the literature. This study demonstrates both the feasibility of compiling and representing this knowledge domain and the possibility of translating it into an efficient automated tool capable of giving reliable expert-like advice at low cost. The reasoning process is divided into three stages. In the first stage, a polymetric composite component is completely defined according to features that are relevant for nondestructive inspection. In the second stage, all the discontinuities that may be present in the component are determined. Finally, in the third stage, appropriate nondestructive testing procedures are identified to detect each of the possible discontinuities.
\end{abstract}

\section{Introduction}

The authors have compiled and formalized the knowledge domain as it appears in the universe of a major automotive manufacturer. The reader should keep in mind that the facts and conclusions unveiled in this work may not be valid for other technical domains where plastic and polymeric composites are used, especially the aerospace industry, where cost, time, and quality constraints and requirements are altogether different.

This work is an attempt to describe the development of a prototype knowledge-based expert system called nondestructive inspection of composite components (NICC), which is intended to:

* Formerly graduate research assistant at the University of Michigan, Ann Arbor. 
1. identify and qualify the potential anomalies that may be present in a given component based on a limited amount of easily obtainable information about that specific part. The analysis considers not only flaws that may be present right after the part has been manufactured but also those that may develop after a short service time, and

2. aid in the selection of the nondestructive inspection techniques that should be specified in order to detect these flaws. Decisions about tests should consider different combinations of requirements such as degree of reliability, time, cost constraints, etc. The system should also give some basic advice on the proper setting of the testing equipment.

Following the development stage characterization outlined by Dym [2], we carried out the following steps when creating the knowledge-based system:

Identification: the problem domain is identified and the goals for the project are set.

Conceptualization: the key attributes of the task and its domain are made explicit and some thought is given to relevant issues of artificial intelligence.

Formalization: a formal model of the task, its attributes, and their relations, are addressed.

Implementation: the tools available for the implementation of the actual system are discussed and the programming of the representation scheme is presented.

Testing: the system is exercised against case studies.

This study addresses polymeric components that are both reinforced and unreinforced. However, the majority of polymeric parts used in cars today have some kind of reinforcing material built into the matrix; hence, this work is focused on composites. From this point on, the term "composite" will be used exclusively when referring to the analyzed materials.

\section{System Characterization and Conceptualization}

A formal description of the task NICC is supposed to perform is:

to determine the flaws that may be present in a given composite component and to determine the nondestructive test methods that should be applied in order to assure its quality.

The basic assumption is that it is possible to determine the nature of flaws that might be present in a given component based on a limited amount of readily available information about this component. After that, with a new set of information about the particular testing situation and environment, one should be able to specify the most effective inspection techniques to assure the quality of the part within the expected time and cost frames and with the necessary accuracy.

To accomplish this task, the reasoning strategy was structured into two parts. First, NICC queries the user in order to collect data about the given 


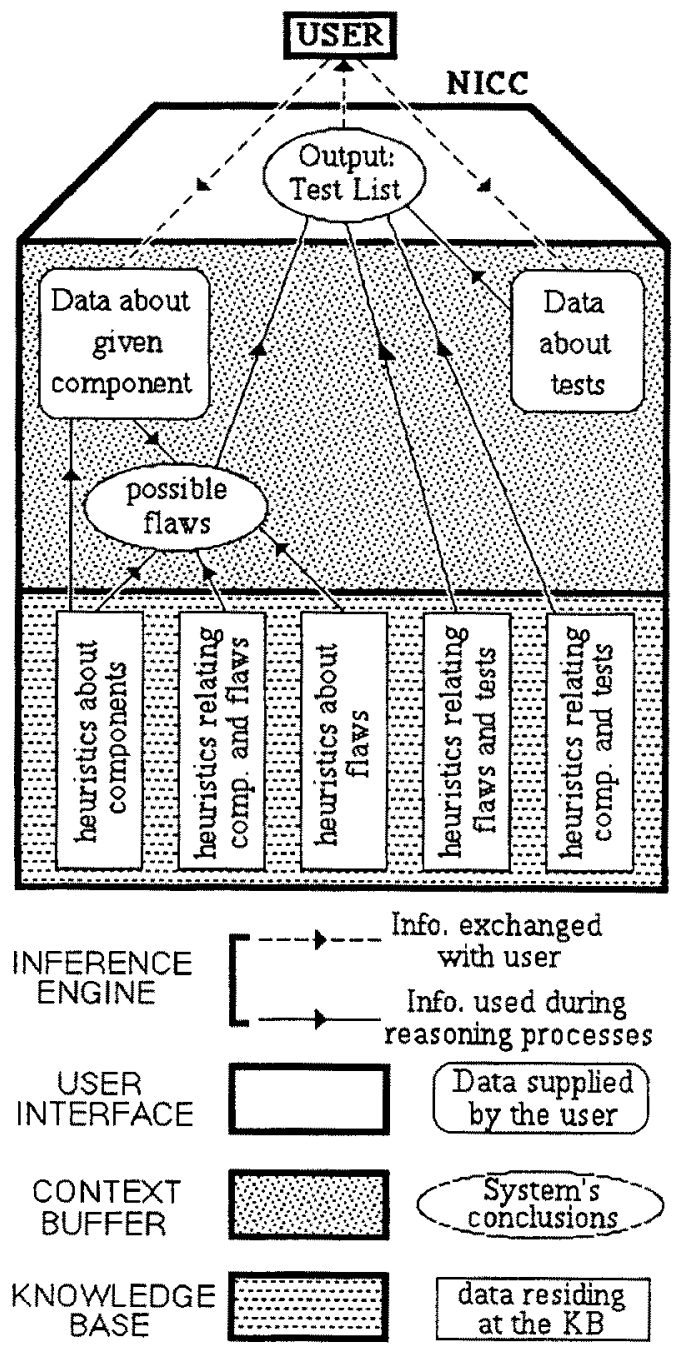

Fig. 1. NICC's architecture.

component (automotive parts, in principle) and uses heuristics (or rules of thumb) and facts from the knowledge base to determine the types of flaws that are likely to occur. Then, after the potential flaws are determined, another set of heuristics is applied. Using another area of the domain knowledge from the knowledge base and some of the facts already established in the first part of the process, the system finally determines the list of tests to be performed, along with basic instructions on the proper settings of the testing machines. This second reasoning process may also request a new set of information from the user, if necessary. The knowledge base must have a priori a complete and updated data bank about all the possible flaws and available test methods, and also the relationships among these objects and the component characteristics. Figure 1 shows a sketch of NICC's reasoning strategy and the data flow. 
It is interesting to note in Fig. 1 that the knowledge base has information about the components in the box "heuristics about components." This means, the system knows beforehand which combination of component parameters is possible and which is not, and is able to guide the users based on their previous answers. So, it is fair to say that there are actually three different steps in the reasoning process, because even before determining any laws, NICC reasons about the characteristics of the components. NICC skips unnecessary questions and allows answers that are consistent with the data already entered. Hence, a completely defined component is, at least in part, also a result of NICC's reasoning.

The type of problem-solving we are dealing with is clearly a diagnostic one. The system will reason about how to classify a complex description of the reality as a node in a diagnostic hierarchy. The search strategy [4] that more adequately suits the task seemed to be backward-chaining (in AI terms), because the number of goal states (the different discontinuities and nondestructive evaluation methods) is much smaller than that of the initial configurations (the hundreds of different combinations of composite components parameters, or parts, that can be used in a car). However, the limited number of possible combinations of the components' characteristics drastically narrows the reasoning path, once the initial nodes are established. Hence, we can say that the branching factor, which is a measurement factor of the search direction with less possible path alternatives, favors a forward-chaining strategy. A best-first search, which is a way to combine the advantages of both depth and breadthfirst search, was definitely the most promising way to explore the problem space. However, the final decision about the search strategy might also be influenced by the nature of the programming environment.

The knowledge representation scheme that seemed most attractive at this first stage was the use of production rules. Other schemes might prove more efficient and could eventually be used in a future system. However, for the development of the prototype system, the advantages offered by a rule-based representation scheme far exceeded the drawbacks. The speech-like format of production rules makes them more easily understood and verifiable by experts, because these are the kind of statements that the experts most frequently use to explain how they do their jobs. Of course, there is some specific information that is not straightforward to obtain using production rules, such as data about the geometry of the component.

\section{Formalization}

\section{Domain Representation}

The efficiency of any methodical procedure designed to analyze large chunks of information is greatly enhanced if the data is handled in a well-organized structure. In order to classify the information that is relevant for the solution-search process, the data was divided into classes of objects. Each object is completely defined by a relatively small number of parameters. Each parameter can have a finite number of different values. This defines a triplet consisting of an object, a 
parameter, and a value. Each triplet object-parameter-value represents a possible state, or node, in the problem space. The problem space is totally defined after all the possible relationships between these nodes are determined. The interaction between the software and the user about any specific component will be then the definition of which nodes should be taken to represent that particular situation and which links should be used to connect these nodes.

The first step in formalizing the problem space will be the complete characterization of all the possible nodes, or object-parameter-value triplets. The second will be the definition of all the possible links, or relationship functions, between them. In this particular problem domain, there are clearly three distinct classes of objects: components, discontinuities, and tests.

For the sake of simplicity of implementation, the first object class was divided into two different subgroups: components $\mathrm{I}$, representing a class of single parts or elemental components, and components II, representing information about bondings. The reasoning behind this division was that, in the case of assemblies or parts that result from the bonding of two or more elemental components, NICC will first check for discontinuities in each of the so-called elemental components individually, whenever applicable. After this procedure is finished, in a subsequent session, NICC will reason about bonding discontinuities alone. Therefore, whenever applicable, each of the components in a bonded assembly should be checked for discontinuities before bonding problems are considered.

Bondings may be of different kinds: adhesive and cohesive. Mechanical bondings are out of the scope of this work and will not be considered. Adhesive bondings imply the use of a third element (the adhesive) between the two components, whereas cohesive bondings are obtained primarily by lamination processes without the use of adhesives. Laminated parts are increasingly finding application in the automotive industry, and in these cases NICC will be investigating the nature of the discontinuities between the laminated layers without actually having reasoned about the individual raw materials that are used in the manufacturing process. The user is expected to know how to differentiate between adhesively bonded parts and laminated sandwiches.

After a careful analysis of the information collected from the experts [1] and from the publications [5, 11-13], and after a detailed evaluation of the reasoning strategies used by the experts to arrive at solutions, a list of attributes for each object class was assembled. These so-called attributes are the characteristics that are relevant for the establishment of a complete solution. A complete solution should determine possible flaws, specify appropriate testing methods, and, when feasible, provide advice on the proper setting of the equipment. It is implicit that if a value is given to each of these parameters for a specific situation, a complete solution can be found. The list of object parameters is given in Table 1.

Table $2 \mathrm{a}$ shows a list of possible values for each of the object parameters of Components I. Tables $2 b, 2 c$, and $2 d$ list values for the objects components II, discontinuities, and tests, respectively. The values given in Table 2 are compiled from information gathered by lengthy discussions with industrial experts [1]. 
Table 1. Lists of object parameters

\begin{tabular}{llll}
\hline \multicolumn{1}{c}{ Components I } & \multicolumn{1}{c}{ Components II } & Discontinuities & \multicolumn{1}{c}{ Tests } \\
\hline Part number & Part number & Type & Name \\
Overall geometry & Adhesive material & Location & Degree of reliability \\
Matrix material & Adherend-near & Size & Capital intensity \\
Manufacturing process & Adherend-far & Orientation & Labor intensity \\
Filler & Application process & & Geometric constraints \\
Presence & Curing process & & Necessity of skilled \\
Material & Relevant geo. features & & operator \\
Reinforcement & Origin & Necessity of safety \\
Presence & Application & & equipment \\
Type & & Location \\
Concentration & & & Application \\
Relevant geo, features & & Instruction flag \\
Origin & & & \\
Application & & & \\
\hline
\end{tabular}

Part number: With this information the system checks the database to determine whether the part has already gone through NICC's consultation. If it did, the system retrieves the result from the data-base. If not, after the session is over, NICC will save its conclusions, making future consultations about the same part more efficient.

Overall geometry: For the sake of simplification we have assumed that any part can be considered basically flat-like or cylindrical-like for local inspection purposes. The implication of this simplifying assumption is that only the local regions of a part that are individually inspected for the occurrence of specific discontinuities are being considered as either flatlike or cylindrical-like.

Reinforcement material: Although there are many recent developments in new materials for reinforcements of plastic parts in the aerospace and racing industries, namely carbon fibers, the automotive industry still uses glass as its primary reinforcement material [1]. We have decided to concentrate our study on glass-reinforced composites and consider its different applications.

Reinforcement concentration: This parameter can only assume two values: low for less than $27 \%$ of reinforcement material in weight or high for concentrations greater than $27 \%$.

Relevant geometric features: Listed under this heading are those features that have an impact on the occurrence of discontinuities and also those that may interfere with the application of certain testing apparatus.

Origin and Application: These two parameters do not have any impact on the occurrence of discontinuities. They are only considered when making decisions about the tests to be recommended. For instance, the presence of a show surface indicates that a portion of the surface of a component may be visible to the customer, and thus problems of aesthetics need to be checked. 
Table 2. Object parameter values
a. Components I
1. Part number
2. Overall geometry
2.1 Flat-like
2.2 Cylinder-like
3. Matrix material
Thermoset resins
3.1 Polyester
3.2 Vinylester
3.3 Epoxy
3.4 Polyurethane
3.5 Polymide
3.6 Phenolic
Thermoplastic resins
3.7 Polyethylene
3.8 Polypropylene
3.9 NYLON
3.10 PEEK (polyether etherketone)
3.11 PPS (polyphenylene sulfide)
3.12 PEI (polyetherimide)
3.13 PAI (polyamideimide)
3.14 PVC (polyvinyl chloride)
3.15 Polystyrene
4. Manufacturing processes
4.1 Lay up
4.2 Injection molding (Thermoplastic)
4.3 Compression molding
4.4 Pultrusion
4.5 Extrusion
4.6 RTM (resin transfer molding)
4.7 RIM (reaction injection molding of Thermosets)
4.8 RRIM (reinforced RIM)
4.9 SRIM (structural RIM)
4.10 Thermo forming
4.11 Blow molding
4.12 Casting
5. Filler
5.1 Presence
5.1.1 Yes
5.1.2 No
5.2 Material
5.2.1 Calcium carbonate
5.2.2 Clay
5.2.3 Mica
5.2.4 Glass microspheres
5.2 .5 Wood flour
5.2.6 Sawdust
5.2.7 Aluminum oxide

6. Reinforcement (glass)

6.1 Presence

6.1.1 Yes

6.1.2 No

6.2 Type

6.2.1 Unidirectionally aligned continuous fibers

6.2.2 Woven continuous fibers

6.2.3 Random continuous fibers

6.2.4 Prefab. mat

6.2.5 Aligned chopped fibers

6.2.6 Random chopped fibers

6.2.7 Particles

6.3 Concentration

6.3.1 Low

6.3.2 High

\section{Origin}

8.1 Normal production

8.2 Special production

8.3 Prototype

8.4 Design study

8.5 After sales

8.6 Environmental exposure

8.7 Vendor evaluation
7. Relevant geometric features

For discontinuities occurrence

7.1 Major changes in cross section

7.2 Ribs on opposite side of show surfaces

7.3 Structural bosses or ribs

7.4 Deep draws

7.5 Sharp corners

7.6 Restricted flow regions

7.7 Wall thickness greater than $4 \mathrm{~mm}$

7.8 Complex geometry

For testing apparatus

7.9 Thickness smaller than $6.4 \mathrm{~mm}$

7.10 Thickness greater than $25.4 \mathrm{~mm}$

7.11 Width smaller than $25.4 \mathrm{~mm}$

7.12 Surface with nonparallel planes

7.13 Any dimension larger than $1.3 \mathrm{~m}$

7.14 Radius of curvature smaller than $15.2 \mathrm{~cm}$
9. Application

9.1 Nominal

9.2 Safety related

9.3 With show surfaces

9.4 Structural/performance 


\section{b. Components II}

1. Part number

4. Adherend-fa

Same as C1-3
2. Adhesive material

2.1 One component epoxy

2.2 Two-component epoxy

2.3 One-component urethane

2.4 Two-component urethane

2.5 Silicon sealant

2.6 Isocyanate

5. Application process

5.1 Manual

5.2 Robotic

5.3 Lamination

\section{Relevant geometric features}

For discontinuities occurrence

7.1 Curved interfaces

7.2 Complex interface coordination

7.3 Joint width smaller than $6.4 \mathrm{~mm}$

For testing apparatus

7.4 Thickness smaller than $6.4 \mathrm{~mm}$

7.5 Thickness greater than $25.4 \mathrm{~mm}$

7.6 Width smaller than $25.4 \mathrm{~mm}$

7.7 Surface with nonparallel planes

7.8 Any dimension larger than $1.3 \mathrm{~m}$

7.9 Radius of curvature smaller than $15.2 \mathrm{~cm}$

\section{c. Discontinuities}

1. Type

1.1 Voids

1.4 Fibers (reinforcement problems)

1.4.1 Misalignment

1.4.2 With poor wet out

1.4.3 Flow lines

1.4.4 Knit lines

1.4.5 Shortage (resin richness)

1.7 Poor conductivity

1.10 Poor surface finishing

1.13 Environmental damage

\section{Location}

2.1 Surface breaking

2.2 Totally embedded

\subsection{Cracks}

1.5 Lack of adhesion

1.5.1 Between fiber and matrix

1.5.2 Between components

1.5.3 Between coating and substrata

1.5.4 Between layers

1.5.4.1 Surface

1.5.4.2 Internal

1.8 Poor coating thickness

1.11 Poor resin cure

1.11.1 Affecting mechanical properties

1.11.2 Affecting geometric properties (warping)

1.14 Service-related flaws*

1.14.1 Declamation

1.14.2 Cracking

1.14.3 Adhesive debonding

3. Size

3.1 Micro

3.2 Small

3.3 Large
3. Adherend-near

Same as CI-3
6. Curing process

6.1 Thermal cure

6.2 Induction heat cure

6.3 Ambient temperature cure

9. Application

Same as C1-9
1.3 Surface pits

1.6 Delaminations

1.6.1 Surface (blister)

1.6.2 Internal
1.9 Surface waviness

1.12 Aesthetical problems

1.12.1 Color mismatch

1.12.2 Blotches

1.12.3 Sink marks

1.12.4 Crazing
4. Orientation

4.1 Parallel

4.2 Perpendicular

(in relation to surface of inspection) 


\begin{tabular}{|c|c|c|}
\hline \multicolumn{3}{|l|}{ d. Tests } \\
\hline $\begin{array}{l}\text { 1. Name } \\
\text { 1.1 Radiation techniques } \\
\text { 1.1.1 Radiographic } \\
\text { 1.1.1.1 Neutron } \\
\text { 1.1.1.2 X-Ray } \\
\text { 1.1.1.2.1 Real time (video) } \\
\text { 1.1.1.2.2 Standard (film) } \\
\text { 1.1.1.2.3 Computerized } \\
\text { tomography } \\
\text { 1.1.2 Beta backscatter }\end{array}$ & $\begin{array}{l}\text { 1.2 Ultrasonic techniques } \\
\text { 1.2.1 Pulse-echo } \\
\text { 1.2.2 Through transmission } \\
\text { 1.2.3 Low frequency } \\
\text { 1.2.4 Acoustic microscopy } \\
\text { 1.2.5 Acoustic } \\
\text { 1.2.6 Back reflection } \\
\text { 1.2.7 Sonic resonants } \\
\text { 1.2.8 Attenuation }\end{array}$ & $\begin{array}{l}\text { 1.3 Liquid penetrants } \\
\text { 1.3.1 Fluorescent } \\
\text { 1.3.2 Visible dye }\end{array}$ \\
\hline $\begin{array}{l}\text { 1.4 Optical techniques } \\
\text { 1.4.1 Proflometry } \\
\text { 1.4.2 Light reflection } \\
\text { 1.4.3 Visual inspection } \\
\text { 1.4.4 Holography }\end{array}$ & $\begin{array}{l}\text { 1.5 Thermographic techniques } \\
\text { 1.5.1 Infrared } \\
\text { 1.5.2 Vibrothermography }\end{array}$ & $\begin{array}{l}\text { 1.6 Acoustic emissions } \\
1.7 \text { Eddy current }\end{array}$ \\
\hline $\begin{array}{l}\text { 2. Degree of reliability } \\
\text { 2.1 Low } \\
\text { 2.2 High }\end{array}$ & $\begin{array}{l}\text { 3. Capital intensity } \\
\text { 3.1 Low } \\
\text { 3.2 High }\end{array}$ & $\begin{array}{l}\text { 4. Labor intensity } \\
\text { 4.1. Low } \\
4.2 \text { High }\end{array}$ \\
\hline $\begin{array}{l}\text { 5. Geometric constraints } \\
5.1 \text { Thickness smaller than } 6.35 \mathrm{~mm} \\
5.2 \text { Thickness greater than } 25.4 \mathrm{~mm} \\
5.3 \text { Width smaller than } 25.4 \mathrm{~mm} \\
5.4 \text { Surfaces with nonparallel plane } \\
5.5 \text { Any dimension larger than } 1.3 \\
5.6 \text { Cylinder like with radius of cur } \\
\text { than } 15.2 \mathrm{~cm}\end{array}$ & are smaller & $\begin{array}{l}\text { 6. Necessity of skilled } \\
\text { operator } \\
6.1 \text { Yes } \\
6.2 \mathrm{No}\end{array}$ \\
\hline $\begin{array}{l}\text { 7. Necessity of safety equipment } \\
7.1 \mathrm{Yes} \\
7.2 \mathrm{No}\end{array}$ & $\begin{array}{l}\text { 8. Location } \\
8.1 \text { Plant } \\
8.2 \text { Supplier }\end{array}$ & \\
\hline 9. Application & 10. Instruction flag & \\
\hline
\end{tabular}

* Due to in-service stresses

\section{The Decision Tree and Node Relationships}

The formalization of the relationship between the values of all the object parameters is the step that will allow the construction of the so-called decision tree, which is the heart of the reasoning scheme used by the system. The decision tree is an acyclic graph where each node represents one particular combination of the triplet object-parameter-value, and each link between these nodes determines the kind of relationship that exists between them. When the system gets closer to completion, all the combinations representing possible states of the problem space should be depicted in this graph.

The solution search process can be thought of as the exploration of the decision tree until one node that matches the goal state is found. There are six different types of links interconnecting nodes. The first three types are internal to the objects (types 1,2 and 3 in Fig. 2). These internal links represent the possible relationships between nodes within a particular class of objects. 


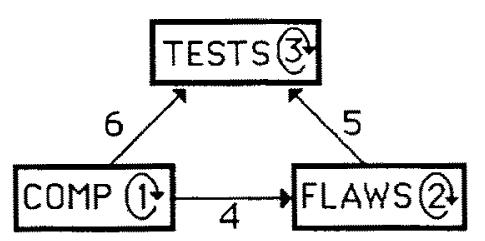

Fig. 2. The six types of links.

Therefore, the system will be able to recognize any inconsistencies in parameters specified by the user. The internal links also make it possible for NICC to determine some values automatically, once a certain combination of values has been defined by the user. For example, internal links of type 1 allow NICC to recognize only the possible combination of parameter values for the object components. For instance, if the user enters a combination of matrix material and manufacturing process that is not feasible, the system will detect the inconsistency and alert the user. Links of type 2 allow the system to make conclusions about discontinuity characteristics based on previous reasoning without unnecessarily questioning the user, whenever possible.

Once the interaction with the user is completed and the component is totally defined, NICC starts its reasoning process to establish the other links that will eventually determine the solution. The other three types of links (see Fig. 2) are the ones that relate completely characterized parts with possible flaw occurrence (link type 4) and with the tests that can be applied to them (type 6), and the links between the flaws and the tests that can detect them (type 5). It is interesting to note that once a possible test is identified NICC might need to query the user to validate links that are internal to the object tests, in order to confirm the indication availability of a particular testing machine.

Links are actually the production rules that compose the knowledge base of the system. Each production rule is associated with one link in the tree, and it can be either the translation of a rule-of-thumb used by an expert or a factual relation, that is, those inter-relations between nodes that are of a scientific nature. Given below are a few sample rules to illustrate the body of the compiled knowledge base.

Rule Base (Links)

a) Internal links

Type 1

\begin{tabular}{llll}
\hline Rule MAT-TYPE-1 & & & \\
If & matrix material & is & polyester \\
Or & matrix material & is & vinylester \\
Or & matrix material & is & epoxy \\
Or & matrix material & is & polyurethane \\
Or & matrix material & is & polymide \\
Or & matrix material & is & phenolic \\
Then & matrix type & is & thermoset resin
\end{tabular}




$\begin{array}{llll}\text { Rule MAT-TYPE-2 } & & \\ \text { If } & \text { matrix material } & \text { is } & \text { polyethylene } \\ \text { Or } & \text { matrix material } & \text { is } & \text { polypropylene } \\ \text { Or } & \text { matrix material } & \text { is } & \text { polystyrene } \\ \text { Or } & \text { matrix material } & \text { is } & \text { NYLON } \\ \text { Or } & \text { matrix material } & \text { is } & P E E K \\ \text { Or } & \text { matrix material } & \text { is } & P P S \\ \text { Or } & \text { matrix material } & \text { is } & P E I \\ \text { Or } & \text { matrix material } & \text { is } & P A I \\ \text { Or } & \text { matrix material } & \text { is } & P V C \\ \text { Then } & \text { material type } & \text { is } & \text { thermoplastic resin }\end{array}$

b) External links

Type 4

$\begin{array}{llll}\begin{array}{l}\text { Rule D4-1 } \\ \text { If }\end{array} & & \\ \text { And } & \text { manuf. process } & \text { is } & \text { compression molding } \\ \text { Then } & \text { rel. geom. feat. } & \text { is } & \begin{array}{l}\text { deep draws } \\ \text { disc. name }\end{array} \\ \text { And } & \text { isc. name } & \text { is } & \text { fibers with poor wet out } \\ \text { Rule D4-2 } & \text { is } & \\ \text { If } & \text { manuf. process } & \text { is } & \text { injection molding } \\ \text { Then } & \text { disc. name } & \text { is } & \text { fiber fiow lines } \\ \text { Rule D4-3 } & \text { manuf. process } & \text { is } & \text { injection molding } \\ \text { If } & \text { disc. name } & \text { is not } & \begin{array}{l}\text { resin richness } \\ \text { delamination }\end{array} \\ \text { Then } & \text { disc. name } & \text { is not } & \end{array}$

The decision tree is the result of the formalization procedure. It is a representation of the knowledge domain that is totally independent of the programming environment or expert system shell. Figure 3 shows a small portion of the decision tree. Numbered boxes are nodes, each with its particular combination of object, parameter, and value (designated by indexes from the lists in object characterization). The optional flag indicate that some interaction with the user is necessary. For example, to validate nodes number 1 and 4, NICC should query the user. The flag in node 5 indicates that a message should be passed to the user alerting that low-frequency ultrasonic equipment requires skilled operators. Links are designated by the letter $L$ followed by a number. Links are represented in the knowledge base by the production rules.

\section{Implementation (GoldWorks II)}

The final version of NICC's working prototype was implemented in GoldWorks II [14]. GoldWorks II allows for the use of different representation schemes, and this feature was used. The objects and their parameters (or slots, using GoldWorks terminology) were implemented as a frame lattice, and the links were represented as production rules.

Figure 4 shows the frame lattice implemented in GoldWorks II. Notice that the top frame is created by GoldWorks II, and it will always remain as the root 


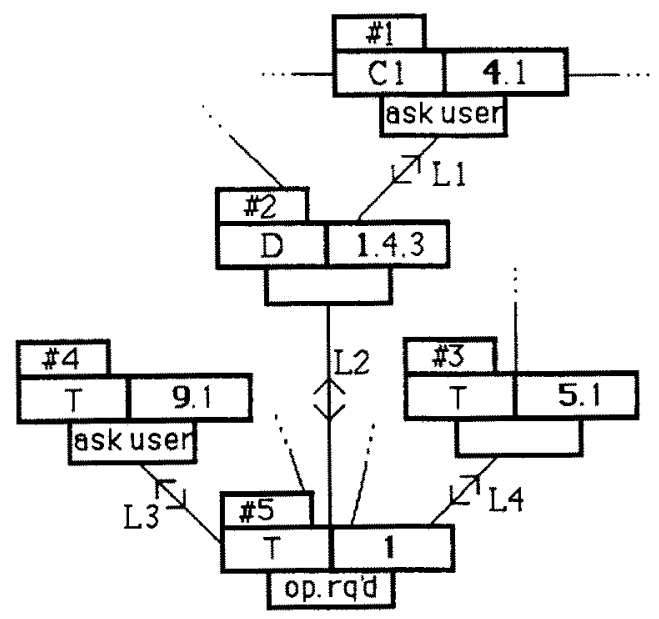

Key $\frac{\frac{\text { node }}{\text { Object } / \mathrm{Par} / \mathrm{Val}}}{\text { [flag }}$

Fig. 3. A section of the decision tree.

of any lattice created within this environment. Its function is to assure that all the frames in the highest working level (those that have the same reasoning hierarchy level) will be part of a single structure. Note that the frame components house the slots that are common to its two child frames, which inherit them.

Figure 4 shows the logical lattice that includes only the objects that are relevant for the reasoning process. The real application lattice is much larger, and it also includes GoldWorks' graphic subsystem. The lists of parameter slots distribution are given in Table 3. Two of the relevant features of the parameter slots are:

Multivalued slots may assume any number of values simultaneously.

Value constraints are optional lists of allowed values for each particular slot. If a list of value constraints is given, the system does not accept values that are different from those specified in the list.

Certainty factors assign levels of certainty or confidence to assertions in the knowledge base. The certainty function provided by GoldWorks II uses a numerical scale ranging from 0.0 to 1.0 , inclusive, where a certainty factor of 1.0 means that the information is known to be true. A certainty factor is not a mathematical expression of probability; rather, it represents a subjective judgment as to the validity of a certain assertion. The system combines the certainties of the assertions that match the antecedent pat- 


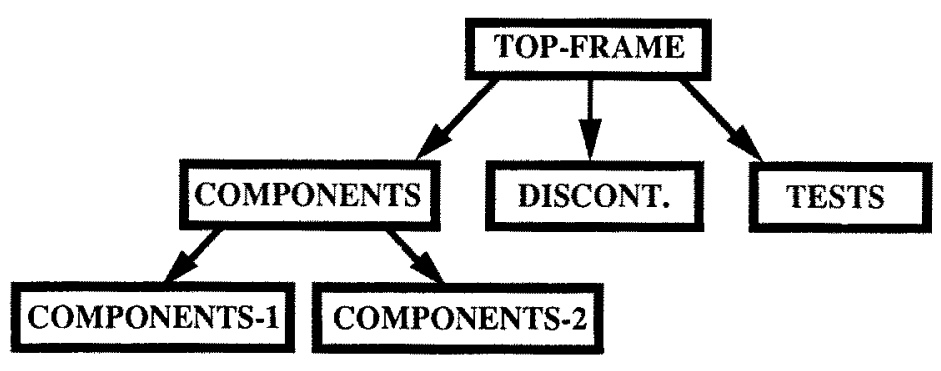

Fig. 4. The object frame structure.

terns of a rule with the certainty factor of the rule itself (which is set when the rule is defined) to calculate the certainty of the new assertion. Table 3 lists values and certainty factors for various objects parameters. The parameter slots housed within the frame components are those that are common to both its child frames, which will inherit them along with the attached values. Although both components I and II have the parameter relevant geometric features, this slot is not placed in the parent frame components because the two lists of associated values are different.

The values for the parameter slots of the discontinuities and tests object classes will be determined by the system's reasoning; hence all the associated default certainty factors will be assumed to be equal to 1.0. The system always attaches an initial default certainty factor to the value of each and every slot. The final certainty of the result, or the system conclusions, will be computed using either the slots default certainty factors or the certainty factor entered by the user. Since this computation is rather slow in the prototype, we only adopt it for the matrix material to demonstrate its use.

GoldWorks provides two different facilities for grouping rules in the rule base: rule sponsors and rule sets. Rule sponsors are used for groups of rules that should be fired in a predetermined sequence. The rules are assigned to sponsors that are arranged in a predetermined sequence. Those rules grouped under the first sponsor will fire before the ones grouped under the second, and so forth. NICC's rule base is organized as follows:

sponsor type-1: rules translating links of type 1, sponsor type-2: rules translating links of types 2 and 4 , sponsor type-3: rules translating links of types 3,5 and 6.

Hence, the rules that determine the component characteristics are fired before those that relate the part to the possible present discontinuities. Finally, totally defined components and associated discontinuities are used by the antecedents of the rules of the last sponsor, which will relate these objects to the appropriate tests.

The second way of grouping rules, that is rule sets, is used when there are mutually exclusive groups of rules. In our case, we have two sets: rules related to components I and rules related to components II. 
Table 3. Values and certainty factors of various object parameters

\section{Components}

Part number

Single valued

Default certainty factor: 1.0

Overall geometry

Single valued

Value constraint: flat like;

cylinder like

Default certainty factor: 1.0

Filler presence

Single valued

Value constraint: yes; no

Default certainty factor: 0.8

Reinforcement type

Single valued

Value constraint: list C1-6.2

Default certainty factor: 0.6

Material type

Single valued

Value constraint: thermoplas-

tic; thermoset

Default certainty factor: 1.0
Origin

Single valued

Value constraint: list $\mathrm{Cl}-8$

Default value: Normal production

Default certainty factor: 1.0

\section{Matrix material}

Single valued

Value constraint: list $\mathrm{C} 1-3$

Default certainty factor: 0.9

Filler material

Single valued

Value constraint: list $\mathrm{C} 1-5.2$

Default certainty factor: 0.5

Reinforcement concentration

Single valued

Value constraint: low; high

Default certainty factor: 0.4
Application

Multivalued

Value constraint: list $\mathrm{Cl}-9$

Default certainty factor: 1.0

\author{
Manufacturing process \\ Single valued \\ Value constraint: list $\mathrm{C1}-4$ \\ Default certainty factor: 1.0
}

Reinforcement presence

Single valued

Value constraint: yes; no

Default certainty factor: 0.8

Relevant geometric features

Multivalued

Value constraint: list $\mathrm{Cl}-7$

Default certainty factor: 1.0

(Note that the parameter material type, even being important information to consider, is not included among the component's characteristics that should be determined from questioning the user, because it is always possible to determine it once the matrix material is known.)

\section{Components II}

Adhesive material

Single valued

Value constraint: list $\mathrm{C} 2-2$

Default certainty factor: 0.8

Application process

Single valued

Value constraint: manual; robotic

Default value: manual

Default certainty factor: 0.9

\section{Discontinuities}

Type

Single valued

Value constraint: list D-1

Default value: none

Size

Single valued

Value constraint: list D-3

Default value: none
Adherend-near

Single valued

Value constraint: list $\mathrm{Cl}-3$

Default certainty factor: 0.9

Curing process

Single valued

Value constraint: list $\mathrm{C} 2-5$

Default value: ambient temp. cure

Default certainty factor: 0.5

Location

Single valued

Value constraint: list D-2

Default value: none

Orientation

Single valued

Value constraint: list D-4

Default value: none
Adherend-far

Single valued

Value constraint: list $\mathrm{Cl}-3$

Default certainty factor: 0.9

Relevant geometric features

Multivalued

Value constraint: list $\mathrm{C} 2-6$

Default certainty factor: 1.0 


\section{Test}

Name

Single valued

Value constraint: list $\mathrm{T}-1$

Default value: none

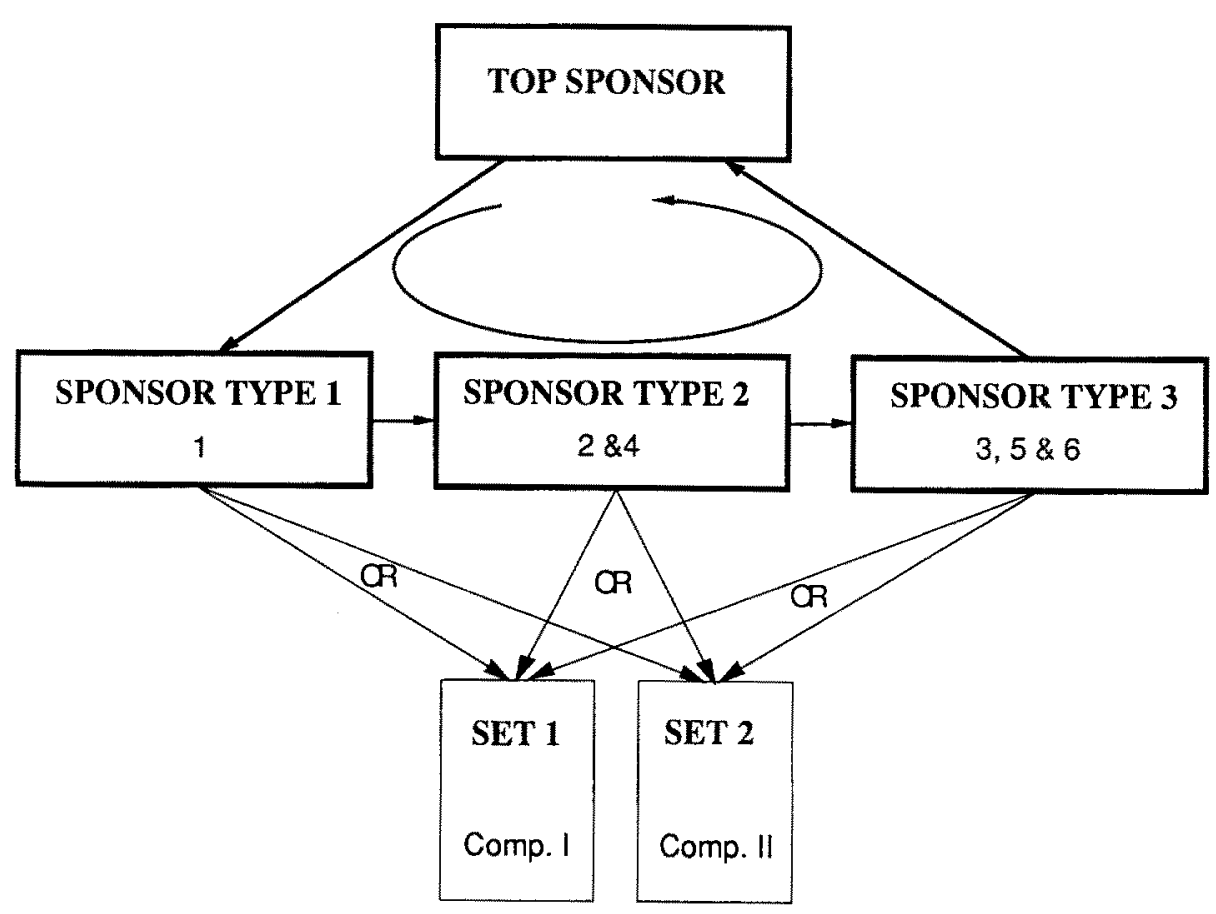

Fig. 5. Sponsors and rule sets arrangement.

Figure 5 shows the arrangement of NICC's rule base and the direction of the consultation loop. It is important to mention at this point that, although GoldWorks provides for the use of either backward, forward or bi-directional rules, NICC's rule base is entirely forward-chained.

Again, top sponsor is a GoldWorks II-created sponsor that serves the same purpose at the top frame in the object frame lattice.

\section{Testing}

As mentioned before, the objective of the implementation part of this work was writing a prototype system. The prototype served the purpose of testing the adopted working methodology. To illustrate the reasoning process, the following values have been chosen: 
Discontinuities

Voids

Cracks

Crazing

Sink marks

Fiber misalignment

Fiber with poor wet out

Fiber flow lines

Fiber knit lines

Resin richness

Internal delaminations

Test Case No. 1

Input (Description of the component)

Application
Origin
Overall geometry
Test site
Filler presence
Filler material
Manufacturing process
Matrix material
Reinforcement presence
Reinforcement type
Relevant geometric features

Tests

Standard X-ray

Real-time X-ray

Ultrasonic pulse echo

Low frequency ultrasonic

Fluorescent penetrant dye

Visual inspection

Infrared thermography

\author{
Nominal \\ Normal production \\ Flat-like \\ Process control \\ Yes \\ Calcium carbonate \\ Injection molding \\ Polyester \\ Yes \\ Random chopped fibers \\ Deep draws \\ Sharp corners \\ Restricted flow regions \\ Thickness smaller than $6.4 \mathrm{~mm}$
}

Note that it was not necessary to enter information about the reinforcement concentration, since reinforced injection molded parts will always have a low reinforcement concentration. It should also be noted that some of the options shown for selection are already the result of NICC's reasoning, based on the user's previous answers. Hence, the importance of the queries sequence.

NICC's inferences on possible discontinuities and recommended NDT methods are listed below.

\section{Discontinuities}

Fiber knit lines

Fibers with poor wet out

Fiber misalignment

Resin richness

Voids
Totally embedded

Parallel

Small

Totally embedded

Parallel

Small \& Iarge

Totally embedded

Parallel

Small \& large

Large

Surface breaking

Small 
Internal delaminations

Crazing

Nondestructive tests

Real-time X-ray

Low frequency ultrasonic

Infrared thermography

Fluorescent liquid penetrant

Visual inspection
Totally embedded

Perpendicular

Small \& large

Surface breaking

Parallel

Note that real-time $X$-ray shows twice in the list; this happens because two different rules resulting in this test fired. It is also interesting to notice that both resin richness and fibers with poor wet out have pulse echo ultrasonic as the first choice for inspection method; however, this technique doesn't show in the indicated test list; this happens because some of the component's characteristics doesn't allow for this kind of inspection. Thus, the second choice should be used.

\section{Conclusions}

This work was not intended to be a final statement on the domain of nondestructive inspections of automotive composite components. It is clearly an initial effort to formalize a rather new domain of knowledge that is still widely scattered and not yet fully understood. The final objective of this research was to prove the feasibility of capturing such knowledge through a prototype system that may serve as a foundation for future development.

Our goal was successfully achieved, because a formal structure for the representation of the knowledge domain of nondestructive testing of plastic and polymeric composite components was presented and translated into a working prototype that, within a constrained universe, arrives at reasonably acceptable results. The expansion of this initial universe is only a matter of following the established framework.

There is still much work left to be done before NICC becomes a full-fledged expert system for NDT. For the most part, the work still necessary to turn this prototype into an operational system is in establishing a complete, or better said, closer to complete, set of production rules or node relationships. Geometric abstractions of the various physical features need further research. Finally, the results obtained by this research were considered very satisfactory by the users [1]. The advantage of using this system is that time and money may be saved by avoiding the application of unnecessary tests to ensure quality.

Acknowledgments. This work was sponsored by Chrysler Corporation, Highland Park, Michigan. The authors would like to thank Mr. Gilbert Chapman II, Pamela Lee and George Harmon of Chrysler Corporation for their inestimable contribution towards successful completion of this research project. 


\section{References}

1. G. Chapman II, G. Harmon, and P. Lee, Chrysler Corporation, Highland Park, Michigan, personal communication (1989/1990)

2. C.L. Dym, AI EDAM 1:37 (1987)

3. P. Harmon and D. King, Systems in Business. Wiley, New York (1985)

4. E. Rich, Artificial Intelligence. McGraw-Hill series in AI (1983)

5. ASM International, Engineered Materials Handbook, vol. 1. Composites. ASM International (1988)

6. D.C. Brown and B. Chandrasekaran, An Approach to Expert Systems for Mechanical Design. Ohio State University, Columbus, OH (1988)

7. J.R. Slagle, M.R. Wick, and M.O. Poliac, AGNESS: A Generalized Network-Based Expert System Shell. University of Minnesota, Minneapolis, MN

8. A. Barr and E.A. Feigenbaum, The Handbook of Artificial Intelligence. Standford University

9. Information Builders, Inc. LEVEL-5 Expert System Software. User's Manual.

10. J.R. Dixon and E.C. Jones, E.C. Libardi, Jr., S.C. Luby, and E.H. Nielsen, Knowledge Representation in Mechanical Design Systems: Issues and Examples. University of Massachusetts, Amherst, MA (1988)

11. G.F. Smith, Fiber Reinforced Plastics in the Automotive Industry. University of Warwick, England (1989)

12. Modern Plastics Encyclopedia 86/87, vol. 63, no. 10A. McGraw-Hill, New York (1987)

13. T.H. Melling, T. Kelly, and P. Walkden, Application of Nondestructive Testing Techniques in the Diagnostics of Faults in Composite Components. Mechanical Engineering Publications Ltd., London (1986)

14. Goldwork II User's Manual. Gold Hill Computers. Boston (1988) 